\title{
Adjuvant radiotherapy in prostate cancer patients with positive margins or extracapsular extension
}

\author{
Chandler Bronkema ${ }^{1,2 \#}$, Nikola Rakic ${ }^{1,2 \#}$, Firas Abdollah $^{1}$ \\ ${ }^{1}$ Vattikuti Urology Institute, Henry Ford Hospital, Detroit, MI, USA; ${ }^{2}$ Wayne State University School of Medicine, Detroit, MI, USA \\ \#These authors contributed equally to this work. \\ Correspondence to: Firas Abdollah. Vattikuti Urology Institute, Henry Ford Hospital, 2799 W Grand Blvd, Detroit, MI 48202, USA. \\ Email: fabdoll1@hfhs.org. \\ Provenance: This is an invited article commissioned by the Section Editor Dr. Peng Zhang (Department of Urology, Zhongnan Hospital of Wuhan \\ University, Wuhan, China). \\ Comment on: Hackman G, Taari K, Tammela TL, et al. Randomised Trial of Adjuvant Radiotherapy Following Radical Prostatectomy Versus Radical \\ Prostatectomy Alone in Prostate Cancer Patients with Positive Margins or Extracapsular Extension. Eur Urol 2019;76:586-95.
}

Submitted Oct 29, 2019. Accepted for publication Nov 07, 2019.

doi: $10.21037 /$ atm.2019.11.44

View this article at: http://dx.doi.org/10.21037/atm.2019.11.44

In men with localized prostate cancer $(\mathrm{PCa})$, radical prostatectomy (RP) is often the treatment of choice and has shown exceptional long-term outcomes $(1,2)$. In patients with adverse pathological features at surgery (positive margins, extracapsular extension, and/or seminal vesicle invasion), randomized trials recommend adjuvant radiotherapy (aRT) to achieve optimal disease control (3-5). In clinical practice, the decision to treat patients with aRT is dependent on several variables, the primary factors including: disease characteristics, degree of added benefit, and risk of unnecessary radiation-related complications. While the role of aRT in patients with adverse pathology at surgery has been extensively explored, the exact benefit in those patients who have some adverse features, but still localized disease, such as a p 2 disease with a positive margin, or $\mathrm{pT} 3 \mathrm{a}$ disease with a negative margin remains to be fully elucidated. In view of this void in recent literature, Hackman et al. reported on the Finnish randomized trial (6).

This report adds to three prior trials within this clinical milieu $(3,5,7)$, in that it is the first to examine the benefit of aRT in patients with $\mathrm{pT} 2$ disease and positive margins or pT3a disease (pNOM0). Hackman et al. found that the 10-year biochemical recurrence-free (BCR-free) survival was $82 \%$ in the adjuvant group and $61 \%$ in the observation group [HR 0.26 (95\% CI, 0.14-0.48), $\mathrm{P}<0.001$ ]. However, this improvement in BCR is not without a cost as these patients displayed higher rates of toxicity. In the aRT arm, there was a higher rate of grade 3 adverse events $(56 \%$ versus $40 \%$ in the observation group, $\mathrm{P}=0.016$ ), which primarily consisted of erectile dysfunction and urinary incontinence. Furthermore, although patients treated with aRT displayed significant benefit in terms of BCRfree survival, these men did not experience improved overall survival (OS) outcomes at 10 years [HR 0.69 (95\% CI, 0.29-1.60), $\mathrm{P}=0.4]$. Such findings that aRT does not provide survival benefit in the subset of patients studied by the current report, and at the same time exposes them to radiation-related complications, should be weighed against the significant risk of $\mathrm{BCR}$ in patients treated with $\mathrm{RP}$ alone.

When appraising the findings of this randomized trial, it is important to view the primary endpoint, BCR-free survival, through an informed lens. BCR-free survival is commonly used as a proxy for OS; however, it has been shown that the two may not indicate interchangeable outcomes. For instance, in 1999, Pound et al. showed that only $34 \%$ of patients with BCR developed metastases suggesting that elevated PSA following RP does not necessarily demonstrate aggressive oncologic progression (8). These findings are further complemented by various studies indicating that in patients with BCR, only certain individuals with poor tumor differentiation and PSA kinetics are at a high risk of disease progression (9-11). Recently, a systematic review of 77 studies analyzed the impact BCR may have on survival, and concluded that in patients who underwent RP and subsequently developed $\mathrm{BRC}$, the main prognostic factor for distant metastases, $\mathrm{PCa}$ 
specific mortality, and overall mortality was a short PSA doubling time (PSA-DT), a high pathologic Gleason Stage $(>8)$, and a short interval to biochemical failure (12). Such findings suggest that the mere presence of BCR following $\mathrm{RP}$ is a relatively insignificant indicator of aggressive disease and survival when compared to PSA-DT, Gleason stage $>8$, and short interval to BCR. The BCR-free survival estimates presented in the present study should be interpreted with this in mind.

While Hackman et al. did not address PSA-DT, or interval to biochemical failure, $94.4 \%$ of patients that were included in the adjuvant arm, and $93.5 \%$ in the observation arm had PCa disease that was Gleason 7 or lower, suggesting that these patients were at low risk of developing distant metastases, PCa specific mortality, and overall mortality. For this subset of patients, BCR may not be quite as sinister as was once thought, especially in the presence of additional disease characteristics such as a lower Gleason score and/or disease $\leq \mathrm{pT} 2$. As such, while statistically significant, the $21 \%$ reduction in BCR between the observation and adjuvant arms, may in fact exaggerate the clinical benefit patients receive from aRT and rushing to treat men with less adverse disease may expose patients to unwarranted toxicity.

As stated previously, Hackman et al. found that $61 \%$ of patients in the observation group were BCR-free at 10 years versus $82 \%$ in the aRT group. Thus, if aRT is widely adopted for these patients, roughly $61 \%$ will incur overtreatment and are at increased risk for radiationrelated complications. For the subset of patients analyzed by Hackman et al., utilization of salvage radiotherapy (sRT) may be more appropriate. Current AUA guidelines recommend sRT administration in men with "PSA or local recurrence after RP in whom there is no evidence of distant metastatic disease" (4). In the present study, 43 patients with protocol-define BCR received sRT, and of these patients, 28 achieved PSA remission and nine required systemic therapy. As stated by the authors, recent data supports the utilization of early sRT (PSA $<0.5 \mathrm{mg} / \mathrm{L}$ ) (13), and in the present study, sRT was given at a cutoff PSA of $0.7 \mathrm{mg} / \mathrm{L}$, which is considered late sRT. It is likely that the implementation of early sRT in the select patients in this study would provide adequate disease management, and at the same time prevent overtreatment in many patients.

Hackman et al. conducted a novel investigation with various strengths. Of particular importance, the present study examined randomized groups representative of contemporary patients (2004 to 2012) and had relatively long median follow-up periods. The median follow-up was 9.3 years in the adjuvant group and 8.6 years in the observation group, indicating that the study relied upon minimal extrapolation for its 10-year BCR-free, overall and metastasis-free survival estimates. Additionally, Hackman et al. collected objective (physician-reported toxicity) and subjective (patient reported quality of life) data to assess the degree of toxicity in these lower risk patients. Despite these strengths, the present study is not devoid of limitations, as stated by the authors. Namely, the study only included a total sample of 250 patients; thus, the small sample size may have impeded its power to fully detect differences in metastases and/or OS, which were secondary endpoints analyzed in this study. To combat this limitation and improve risk stratification in patients whom benefit most from aRT, additional tools may be necessary, a propitious option being genetic classification. Recent literature has shown genetic classification modalities (Prolaris, Oncotype $\mathrm{Dx}$, and Decipher) in conjunction with clinicopathologic variables to be promising in predicting metastasis in $\mathrm{RP}$ patients and helpful in decision making of aRT versus sRT (14-16). Hackman et al. found that in these lower-risk patients, there were $2 \mathrm{PCa}$-related deaths (one in each arm), six patients (two in the aRT arm and four in the observation arm) developed metastasis, and nine were castration resistant (three in aRT group and six in the observation group). Genetic classification methods could certainly be advantageous in risk stratifying this population of men studied by Hackman et al., to further aid in the proactive selection of patients with unfavorable outcomes who would benefit from aRT, further preventing overtreatment in patients with less aggressive disease.

In all, the report by Hackman et al. is an important contribution to recent literature as it answers questions about the role of aRT in a subset of PCa patients for which the benefit of aRT still needs to be conclusively defined. In view of the present study findings, treatment of all patients with surgical margins and extracapsular extension with aRT poses a considerable risk of unnecessary treatment, and at the same time does not provide a survival and metastatic benefit. Although Hackman et al. displayed that aRT improves BCR-free survival when compared to initial observation, the significance of this discovery should be kept in perspective and considered in juxtaposition with other endpoints like OS, PCa-specific survival and metastasis-free survival. In addressing this conflict of overtreatment versus disease control, genomic classification and sRT prove to be promising options in optimally selecting patients that benefit 
from aRT and reducing unnecessary radiation toxicity, respectively. We look forward to future explorations that complement the conclusions presented by Hackman et al. and continue to optimize clinical management in $\mathrm{PCa}$ patients.

\section{Acknowledgments}

None.

\section{Footnote}

Conflicts of Interest: The authors have no conflicts of interest to declare.

Ethical Statement: The authors are accountable for all aspects of the work in ensuring that questions related to the accuracy or integrity of any part of the work are appropriately investigated and resolved.

\section{References}

1. Hamdy FC, Donovan JL, Lane JA, et al. 10-year outcomes after monitoring, surgery, or radiotherapy for localized prostate cancer. N Engl J Med 2016;375:1415-24.

2. Mottet N, Bellmunt J, Bolla M, et al. EAU-ESTROSIOG Guidelines on Prostate Cancer. Part 1: Screening, Diagnosis, and Local Treatment with Curative Intent. Eur Urol 2017;71:618-29.

3. Bolla M, van Poppel H, Collette L, et al. Postoperative radiotherapy after radical prostatectomy: a randomised controlled trial (EORTC trial 22911). Lancet 2005;366:572-8.

4. Thompson IM, Valicenti RK, Albertsen P, et al. Adjuvant and salvage radiotherapy after prostatectomy: AUA/ ASTRO Guideline. J Urol 2013;190:441-9.

5. Wiegel T, Bottke D, Steiner U, et al. Phase III postoperative adjuvant radiotherapy after radical prostatectomy compared with radical prostatectomy alone in $\mathrm{pT} 3$ prostate cancer with postoperative undetectable prostate-specific antigen: ARO 96-02/AUO AP 09/95. J Clin Oncol 2009;27:2924-30.

6. Hackman G, Taari K, Tammela TL, et al. Randomised Trial of Adjuvant Radiotherapy Following Radical Prostatectomy Versus Radical Prostatectomy Alone in Prostate Cancer Patients with Positive Margins or Extracapsular Extension. Eur Urol 2019;76:586-95.

7. Thompson IM, Tangen CM, Paradelo J, et al. Adjuvant radiotherapy for pathological T3N0M0 prostate cancer significantly reduces risk of metastases and improves survival: long-term followup of a randomized clinical trial. J Urol 2009;181:956-62.

8. Pound CR, Partin AW, Eisenberger MA, et al. Natural history of progression after PSA elevation following radical prostatectomy. JAMA 1999;281:1591-7.

9. Antonarakis ES, Feng Z, Trock BJ, et al. The natural history of metastatic progression in men with prostatespecific antigen recurrence after radical prostatectomy: long-term follow-up. BJU Int 2012;109:32-9.

10. van den Bergh RC, van Casteren NJ, van den Broeck T, et al. Role of Hormonal Treatment in Prostate Cancer Patients with Nonmetastatic Disease Recurrence After Local Curative Treatment: A Systematic Review. Eur Urol 2016;69:802-20.

11. Brockman JA, Alanee S, Vickers AJ, et al. Nomogram Predicting Prostate Cancer-specific Mortality for Men with Biochemical Recurrence After Radical Prostatectomy. Eur Urol 2015;67:1160-7.

12. Van den Broeck T, van den Bergh RCN, Arfi N, et al. Prognostic Value of Biochemical Recurrence Following Treatment with Curative Intent for Prostate Cancer: A Systematic Review. Eur Urol 2019;75:967-87.

13. Fossati N, Karnes RJ, Boorjian SA, et al. Long-term Impact of Adjuvant Versus Early Salvage Radiation Therapy in pT3N0 Prostate Cancer Patients Treated with Radical Prostatectomy: Results from a Multi-institutional Series. Eur Urol 2017;71:886-93.

14. Fine ND, LaPolla F, Epstein M, et al. Genomic classifiers for treatment selection in newly diagnosed prostate cancer. BJU International 2019. [Epub ahead of print]

15. Gore JL, du Plessis M, Santiago-Jiménez M, et al. Decipher test impacts decision making among patients considering adjuvant and salvage treatment after radical prostatectomy: Interim results from the Multicenter Prospective PROIMPACT study. Cancer 2017;123:2850-9.

16. Dalela D, Santiago-Jiménez M, Yousefi K, et al. Genomic Classifier Augments the Role of Pathological Features in Identifying Optimal Candidates for Adjuvant Radiation Therapy in Patients With Prostate Cancer: Development and Internal Validation of a Multivariable Prognostic Model. J Clin Oncol 2017;35:1982-90.

Cite this article as: Bronkema C, Rakic N, Abdollah F. Adjuvant radiotherapy in prostate cancer patients with positive margins or extracapsular extension. Ann Transl Med 2019;7(Suppl 8):S291. doi: 10.21037/atm.2019.11.44 